International Journal of

Environmental Research and

Public Health

ISSN 1660-4601

www.mdpi.com/journal/ijerph

Article

\title{
Resistance of Stenotrophomonas maltophilia to Fluoroquinolones: Prevalence in a University Hospital and Possible Mechanisms
}

\author{
Wei Jia ${ }^{1, *}$, Jiayuan Wang ${ }^{2}$, Haotong $\mathrm{Xu}^{2}$ and Gang $\mathrm{Li}^{1}$
}

1 Medical Experimental Center, General Hospital of Ningxia Medical University, 804 Shengli Street, Yinchuan City, Ningxia Hui Autonomous Region 750004, China; E-Mail: gone.lee@163.com

2 School of Laboratory Medicine, Ningxia Medical University, 1160 Shengli Street, Yinchuan City, Ningxia Hui Autonomous Region 750004, China; E-Mails: wjy10816170105@163.com (J.W.); xht304@126.com (H.X.)

* Author to whom correspondence should be addressed; E-Mail: jiawei6365@126.com; Tel./Fax: +86-951-674-3322.

Academic Editor: Paul B. Tchounwou

Received: 16 March 2015 / Accepted: 4 May 2015 / Published: 13 May 2015

\begin{abstract}
Objective: The purpose of this study was to investigate the clinical distribution and genotyping of Stenotrophomonas maltophilia, its resistance to antimicrobial agents, and the possible mechanisms of this drug resistance. Methods: S. maltophilia isolates were collected from clinical specimens in a university hospital in Northwestern China during the period between 2010 and 2012, and were identified to the species level with a fully automated microbiological system. Antimicrobial susceptibility testing was performed for S. maltophilia with the Kirby-Bauer disc diffusion method. The minimal inhibitory concentrations $\left(\mathrm{MIC}_{\mathrm{s}}\right)$ of norfloxacin, ofloxacin, chloramphenicol, minocycline, ceftazidime, levofloxacin and ciprofloxacin against $S$. maltophilia were assessed using the agar dilution method, and changes in the MIC of norfloxacin, ciprofloxacin and ofloxacin were observed after the addition of reserpine, an efflux pump inhibitor. Fluoroquinolone resistance genes were detected in $S$. maltophilia using a polymerase chain reaction (PCR) assay, and the expression of efflux pump smeD and $s m e F$ genes was determined using a quantitative fluorescent (QF)-PCR assay. Pulsed-field gel electrophoresis (PFGE) was employed to genotype identified S. maltophilia isolates. Results: A total of 426 S. maltophilia strains were isolated from the university hospital from 2010 to 2012, consisting of $10.1 \%$ of total non-fermentative bacteria. The prevalence of norfloxacin,
\end{abstract}


ciprofloxacin and ofloxacin resistance was $32.4 \%, 21.9 \%$ and $13.2 \%$ in the $114 \mathrm{~S}$. maltophilia isolates collected from 2012, respectively. Following reserpine treatment, $19 \mathrm{~S}$. maltophilia isolates positive for efflux pump were identified, and high expression of $s m e D$ and $s m e F$ genes was detected in two resistant isolates. gyrA, parC, smeD, smeE and smeF genes were detected in all $114 \mathrm{~S}$. maltophilia isolates, while smqnr gene was found in $25.4 \%$ of total isolates. Glu-Lys mutation (GAA-AAA) was detected at the 151th amino acid of the gyrA gene, while Gly-Arg mutation (GGC-CGC) was found at the 37th amino acid of the $\operatorname{par} C$ gene. However, no significant difference was observed in the prevalence of gyr $A$ or parC mutation between fluoroquinolone-resistant and -susceptible isolates $(p>0.05)$. The smqnr gene showed $92 \%$ to $99 \%$ heterogenicity among the 14 S. maltophilia clinical isolates. PFGE of 29 smqnr gene-positive $S$. maltophilia clinical isolates revealed 25 PFGE genotypes and 28 subgenotypes. Conclusions: Monitoring the clinical distribution and antimicrobial resistance of $S$. maltophilia is of great significance for the clinical therapy of bacterial infections. Reserpine is effective to inhibit the active efflux of norfloxacin, ciprofloxacin and ofloxacin on S. maltophilia and reduce MIC of fluoroquinolones against the bacteria. The expression of efflux pump smeD and $s m e F$ genes correlates with the resistance of $S$. maltophilia to fluoroquinolones.

Keywords: Stenotrophomonas maltophilia; antimicrobial resistance; reserpine; fluoroquinolone; pulsed-field gel electrophoresis

\section{Introduction}

Stenotrophomonas maltophilia, an aerobic, non-fermentative bacterium, is predominantly found in immunocompromised individuals and those receiving long-term, large-dose broad-spectrum antimicrobial agents [1-3]. This bacterium, which frequently colonizes medical devices, may induce catheter-associated infections, bacteriaemia, urinary infections, and infections at other multiple sites [4,5]. Among the non-fermentative bacteria, S. maltophilia ranks third to Pseudomonas aeruginosa and Acinetobacter spp. in the prevalence among clinical isolates [1]. The natural resistance to imipenem and high resistance to multiple clinically commonly used antimicrobial agents leave few antimicrobial options for this species, making treatment of patients infected with S. maltophilia very difficult [6-8]. Fluoroquinolones are a class of chemicals that are effective for the treatment of infections caused by $S$. maltophilia infections [9-11], however, there is an increasing reported prevalence of resistance to fluoroquinolones in S. maltophilia [12-14]. It is reported that the resistance of $S$. maltophilia to fluoroquinolones may be mainly caused by the mutation at the target sites of DNA gyrase and topoisomerase [15-18], plasmid or chromosome-mediated mutations of drug resistance genes [19,20] and drug efflux pumps [21-23]. Since multiple mechanisms are found to be involved in the development of resistance to fluoroquinolones in S. maltophilia, an increasing prevalence of fluoroquinolone resistance is detected in S. maltophilia. Understanding of the mechanisms underlying fluoroquinolone resistance may therefore be helpful to interrupt and prevent the emergence of antibiotic-resistant $S$. maltophilia and reduce the prevalence and mortality of $S$. maltophilia infections. In this study, we investigated the distribution and 
antimicrobial resistance of 426 clinical strains of S. maltophilia isolated during the period between 2010 and 2012from a university hospital located in Northwestern China, and explored the possible mechanisms of the observed resistance, so as to provide evidence as a basis for the inappropriate clinical use of antimicrobial agents and the control and prevention of $S$. maltophilia infections. In addition, pulsed-field gel electrophoresis (PFGE) was used for genotyping clinical isolates of S. maltophilia to understand the characteristics of hospital-acquired infections of this bacterium.

\section{Materials and Methods}

\subsection{S. maltophilia Isolates}

During the period from 2010 through 2012, a total of 426 clinical isolates of S. maltophilia were collected from various clinical specimens in a university hospital in Northwestern China. All isolates were identified to species level with a VITEK-2 COMPACT fully automated microbiological system (BioMérieux, Inc.; Durham, NC, USA).

\subsection{Determination of Minimum Inhibitory Concentration of Fluoroquinolones}

The minimum inhibitory concentration (MIC) of seven fluoroquinolones including norfloxacin, ofloxacin, chloramphenicol, minocycline, ceftazidime, levofloxacin, and ciprofloxacin (National Institutes for Food and Drug Control, Beijing, China; all potency $>95 \%$ ), on S. maltophilia was estimated using the agar dilution method [24], and was assessed according to the Clinical Laboratory Standard Institute (CLSI) published interpretive criteria, while Escherichia coli ATCC25922 (Shanghai Harmony Biotechnology Co., Ltd.; Shanghai, China) served as quality control bacterial strains.

\subsection{Effect of Reserpine on Fluoroquinolone MIC}

The Mueller-Hinton (M-H) agar medium (Oxoid, Basingstoke, United Kingdom) containing norfloxacin, ciprofloxacin, and ofloxacin at a 1:2 dilution was added with an efflux pump inhibitor reserpine (Dalian Meilun Biology Technology Co., Ltd.; Dalian, China) at a final concentration of $20 \mathrm{mg} / \mathrm{L}$ to determine the MIC of $S$. maltophilia to these three fluoroquinolones in the presence of efflux pump inhibitor, while the medium treated with reserpineat the same concentration served as controls. The change in the MIC of norfloxacin, ciprofloxacin, and ofloxacin on S. maltophilia was evaluated before and after reserpine treatment, and a MIC reduction of $1 / 4$ or less was considered efflux pump positive $[25,26]$.

\subsection{Detection of Fluoroquinolone Resistance Genes in S. maltophilia Clinical Isolates}

Genomic DNA was isolated from S. maltophilia colonies using an Invitrogen ${ }^{\mathrm{TM}}$ genomic DNA extraction kit, and fluoroquinolone resistance genes were detected in the 114 clinical isolates of S. maltophilia using a polymerase chain reaction (PCR) assay with the primers (Table 1) synthesized by the Sangon Biotech (Shanghai) Co., Ltd. (Shanghai, China), including qnrA, qnrB, qnrS, qnrC, qnrD, aac-lb-Cr, qepA, oqxA, oqxB, smeE, smeF, smeD, gyrA, parC and smqnr [27-30]. PCR was performed with a $25 \mu \mathrm{L}$ system containing $1 \mu \mathrm{L}$ template DNA, $1 \mu \mathrm{L}$ of the forward and reverse primers, 


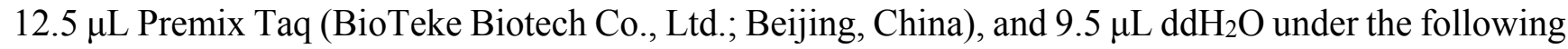
conditions: pre-degeneration at $95{ }^{\circ} \mathrm{C}$ for $5 \mathrm{~min}$, followed by 30 cycles of degeneration at $95{ }^{\circ} \mathrm{C}$ for $30 \mathrm{~s}$, annealing at the temperature shown in Table 1 for $30 \mathrm{~s}$, and extension at $72{ }^{\circ} \mathrm{C}$ for $1 \mathrm{~min}$, and final extension at $72{ }^{\circ} \mathrm{C}$ for $7 \mathrm{~min}$. The amplification products were checked on a $1.5 \%$ agarose gel by electrophoresis.

Table 1. PCR primer sequence and PCR product size.

\begin{tabular}{|c|c|c|c|}
\hline $\begin{array}{l}\text { Fluoroquinolone } \\
\text { Resistance Gene }\end{array}$ & Sequence & $\begin{array}{l}\text { Annealing } \\
\text { Temperature }\left({ }^{\circ} \mathrm{C}\right)\end{array}$ & $\begin{array}{l}\text { Product } \\
\text { Size (bp) } \\
\end{array}$ \\
\hline$q n r A$ & $\begin{array}{l}\text { F: 5'-AGAGGATTTCTCACGCCAGG-3'; } \\
\text { R: 5'-TGCCAGGCACAGATCTTGAC-3' }\end{array}$ & 63 & 580 \\
\hline$q n r B$ & $\begin{array}{l}\text { F: 5'-GGMATHGAAATTCGCCACTG-3'; } \\
\text { R: 5'- TTTGCYGYYCGCCAGTCGAA }\end{array}$ & 56 & 264 \\
\hline$q n r S$ & $\begin{array}{l}\text { F: 5'-GCAAGTTCATTGAACAGGGT-3'; } \\
\text { R: 5'-TCTAAACCGTCGAGTTCGGCG-3' }\end{array}$ & 56 & 428 \\
\hline$q n r C$ & $\begin{array}{l}\text { F: 5'-GGGTTGTACATTTATTGAATCG-3'; } \\
\text { R: 5'-CACCTACCCATTTATTTTCA-3' }\end{array}$ & 56 & 310 \\
\hline$q n r D$ & $\begin{array}{l}\text { F: 5'-GGGTTGATTTAACTGATAC-3'; } \\
\text { R: 5'-TTCGCACTTTTCTAATATGAC-3' }\end{array}$ & 56 & 310 \\
\hline$a a c-I b-C r$ & $\begin{array}{l}\text { F: 5'-TTGCGATGCTCTATGAGTGGCTA-3'; } \\
\text { R: 5'-CTCGAATGCCTGGCGTGTTT-3' }\end{array}$ & 58 & 482 \\
\hline qepA & $\begin{array}{l}\text { F: 5'-GCAGGTCCAGCAGCGGGTAG-3'; } \\
\text { R: 5'-CTTCCTGCCCGAGTATCGTG-3' }\end{array}$ & 48 & 199 \\
\hline oqxA & $\begin{array}{l}\text { F: 5'-CTTGCACTTAGTTAAGCGCC-3'; } \\
\text { R: 5'-GAGGTTTTGATAGTGGAGGTAGG-3' }\end{array}$ & 65 & 866 \\
\hline$o q x B$ & $\begin{array}{l}\text { F: 5'-GCGGTGCTGTCGATTTTA-3'; } \\
\text { R: 5'- TACCGGAACCCATCTCGAT-3' }\end{array}$ & 65 & 781 \\
\hline smeE & $\begin{array}{l}\text { F: 5'-AGCTCGACGCCACGGTA-3'; } \\
\text { R: 5'- TGGCCTGGATCGAGAGCA-3' }\end{array}$ & 55 & 803 \\
\hline smeF & $\begin{array}{l}\text { F: 5'-GCCACGCTGAAGACCTA-3'; } \\
\text { R: 5'-CACCTTGTACAGGGTGA-3' }\end{array}$ & 55 & 800 \\
\hline smeD & $\begin{array}{l}\text { F: 5'-CCAAGAGCCTTTCCGTCAT-3'; } \\
\text { R: 5'- TCTCGGACTTCAGCGTGAC-3' }\end{array}$ & 58 & 150 \\
\hline gyrA & $\begin{array}{l}\text { F: 5'-AACTCAACGCGCACAGCAACAAGCC-3'; } \\
\text { R: 5'-CCAGTTCCTTTTCGTCGTAGTTGGG-3' }\end{array}$ & 58 & 300 \\
\hline parC & $\begin{array}{l}\text { F: 5'-ATCGGCGACGGCCTGAAGCC-3'; } \\
\text { R: 5'-CGGGATTCGGTATAACGCAT-3' }\end{array}$ & 55 & 273 \\
\hline$s m q n r$ & $\begin{array}{l}\text { F: 5'-GCTCTAGAGCTCTACGAATGCGATTTCTCCG-3'; } \\
\text { R: 5'- CGGAATTCCGAAACTGGCACCGCTCACG-3' }\end{array}$ & 52 & 817 \\
\hline gyrA* & $\begin{array}{l}\text { F: 5'-AACTCAACGCGCACAGCAACAAGCC-3'; } \\
\text { R: 5'-CCAGTTCCTTTTCGTCGTAGTTGGG-3' }\end{array}$ & 58 & 300 \\
\hline$s m e D^{*}$ & $\begin{array}{l}\text { F: 5'-CCAAGAGCCTTTCCGTCAT-3'; } \\
\text { R: 5'- TCTCGGACTTCAGCGTGAC-3' }\end{array}$ & 58 & 150 \\
\hline$s m e F^{*}$ & $\begin{array}{l}\text { F: 5'-CCAACGCGGATCGTGATATC-3'; } \\
\text { R: 5'-TGCTCATCCAGGCTGACATTC-3' }\end{array}$ & 58 & 100 \\
\hline
\end{tabular}


Following electrophoresis for $30 \mathrm{~min}$, the agarose gel was stained with ethidium bromide $(0.5$ $\mu \mathrm{g} / \mathrm{mL}$ ), and then visualized with a gel imaging analysis system. The target DNA fragment was sequenced by the Beijing Sunbiotech Co., Ltd. (Beijing, China), and aligned to the sequences released in GenBank.

\subsection{Quantitative Fluorescent $(Q F)-P C R$}

A total of six S. maltophilia isolates were used for QF-PCR assay, including three randomly selected efflux pump-positive isolates that were resistant to fluoroquinolones, one randomly selected efflux pump-negative isolate that was resistant to fluoroquinolone, one efflux pump-negative isolate that was sensitive to norfloxacin, ciprofloxacin, and ofloxacin, and one S. maltophilia ATCC13637 isolate (Shanghai Harmony Biotechnology Co., Ltd.) that served as an efflux pump-negative control isolate. Total RNA was isolated from $S$. maltophilia isolates using the AxyPrep RNA extraction kit (Axygen Biosciences, Inc.; Union City, CA, USA) following the manufacturer's instructions.

Total RNA was reversely transcribed into cDNA using a cDNA reverse transcription kit (Beijing TransGen Biotech Co., Ltd.; Beijing, China) according to the manufacturer's instructions. Briefly, cDNA was synthesized in a $20 \mu \mathrm{L}$ reaction system containing $1 \mu \mathrm{g}$ RNA, $1 \mu \mathrm{L}$ reverse primer, $10 \mu \mathrm{L} 2 \times \mathrm{TS}$ Reaction Mix, $1 \mu \mathrm{L}$ RT Enzyme Mix, $1 \mu \mathrm{L}$ gDNA Remover and $7 \mu \mathrm{L}$ RNase-free water under the following condition: at $25{ }^{\circ} \mathrm{C}$ for $10 \mathrm{~min}$, at $42{ }^{\circ} \mathrm{C}$ for $30 \mathrm{~min}$, and at $85{ }^{\circ} \mathrm{C}$ for $5 \mathrm{~min}$. The mRNA expression of smeD and smeF genes in S. maltophilia isolates was detected using a QF-PCR assay with the primers (Table 1) synthesized by the Sangon Biotech (Shanghai) Co., Ltd. [31,32] on a Roche Light cycler 480 PCR System (Roche, Basel, Switzerland), and gyrA served as an internal reference gene. The PCR assays were performed by pre-denaturation for $30 \mathrm{~s}$ at $95{ }^{\circ} \mathrm{C}$, followed by 45 cycles of $5 \mathrm{~s}$ at $95{ }^{\circ} \mathrm{C}$ and $30 \mathrm{~s}$ at $58{ }^{\circ} \mathrm{C}$. Melting curve analysis was also used to check the specificity of the amplification reaction. $\mathrm{ddH}_{2} \mathrm{O}$, as alternative of template cDNA, was used as a negative control. Relative quantity of $s m e D$ and $s m e F m R N A$ expression was calculated by using the $2^{-\Delta \Delta \mathrm{CT}}$ method, while the relative expression of gyrA $m R N A$ in the S. maltophilia ATCC13637 isolate was defined as 1 .

\section{6. $P F G E$}

PFGE was performed in 29 S. maltophilia clinical isolates that were positive for smqnr gene and one quality control isolate Salmonella typhi H9812. Briefly, single colony was added with $20 \mu \mathrm{L}$ lysozyme (20 mg/mL; Beijing TransGen Biotech Co., Ltd.), placed in water bath at $55{ }^{\circ} \mathrm{C}$ for $15 \mathrm{~min}$, and then added with $20 \mu \mathrm{L}$ protease $\mathrm{K}(20 \mathrm{mg} / \mathrm{mL}$; Beijing TransGen Biotech Co., Ltd.). Then, $400 \mu \mathrm{L}$ agarose (1\%) with a low melting point was added, mixed, and frozen. The mixture was transferred to $1 \mathrm{~mL}$ lysozyme buffer and $5 \mu \mathrm{L}$ protease $\mathrm{K}(20 \mathrm{mg} / \mathrm{mL})$, placed at a water bath at $55{ }^{\circ} \mathrm{C}$ for $2 \mathrm{~h}$, and then the supernatant was discarded. The sediment was rinsed twice with pre-warmed $\left(54{ }^{\circ} \mathrm{C}\right)$ pure water, and washed three times with pre-warmed $\left(54{ }^{\circ} \mathrm{C}\right) 1 \times \mathrm{TE}$ buffer, of 10 min each time. The gel was cleaved with $100 \mu \mathrm{L}$ restriction enzyme $X b a I$ on a water batch at $37^{\circ} \mathrm{C}$ for $2 \mathrm{~h}$. Finally, electrophoresis was performed in $1 \times \mathrm{TBE}$ buffer at $14^{\circ} \mathrm{C}, 6 \mathrm{~V} / \mathrm{cm}, 120^{\circ}$ angular field of view for $22 \mathrm{~h}$. Pulse time was increased linearly from $5 \mathrm{~s}$ initially to $35 \mathrm{~s}$ finally during the run.Following electrophoresis for $30 \mathrm{~min}$, the agarose gel was stained with ethidium bromide $(0.5 \mu \mathrm{g} / \mathrm{mL})$, and then visualized with a gel imaging analysis system. The target DNA fragment was sequenced by the 
Beijing Sunbiotech Co., Ltd. PFGE patterns were interpreted according to the criteria suggested by Tenover et al. [33].

\subsection{Statistics}

All data were processedin the software WHONET version 5.6, and all statistical analyses were done with the software SPSS version 11.5 (SPSS Inc.; Chicago, IL, USA). The differences of proportions were tested for statistical significance with chi-square test, while Student $t$ test was employed to compare between groups. A $p$ value $<0.05$ was considered statistically significant.

\section{Results}

\subsection{Prevalence of S. maltophilia clinical Isolates}

A total of 426 S. maltophilia strains were isolated from the university hospital from 2010 to 2012 , consisting of $10.1 \%(426 / 4225)$ of total non-fermentative bacteria and $1.8 \%(426 / 23,880)$ of total clinical bacterial isolates, and the prevalence of $S$. maltophilia ranked third to P. aeruginosa and A. baumannii and sixth in all Gram-negative bacilli. Of the 426 S. maltophilia clinical isolates, $83.3 \%$ were isolated from sputum and throat swab, $4.5 \%$ from catheters andexcreta, $1.6 \%$ from blood, and $7.1 \%$ from cerebrospinal fluid, sterile body fluid and other samples.

\subsection{Resistance of S. maltophilia to Fluoroquinolones}

The 426 clinical isolates of S. maltophilia showed the highest susceptibility to minocycline, with a $0.5 \%$ prevalence of minocycline resistance detected, while $3.3 \%$ and $74.3 \%$ of the isolates were resistant to levofloxacin and sulfamethoxazole, respectively. During the study period from 2010 to 2012, no significant differences were detected in the prevalence of minocycline or levofloxacin resistance in S. maltophilia clinical isolates (all $p$ values $>0.05$ ); however, there was a significant rise seen in the prevalence of sulfamethoxazole resistance $\left(\chi^{2}=36.963, p<0.01\right)$. In addition, the prevalence of resistance to norfloxacin, ciprofloxacin, and ofloxacin appeared a rise tendency in S. maltophilia clinical isolates during the study period (Table 2).

\subsection{Fluoroquinolone Resistance in S. maltophilia Isolated from Various Departments of the Hospital}

The prevalence of fluoroquinolone resistance varied in S. maltophilia isolated from different departments of the hospital (Table 3). A higher prevalence (95\%) of sulfamethoxazole resistance was detected in S. maltophilia isolated from the Department of Neurology than from the Intensive Care Unit (ICU), Department of Respiratory Medicine, and Department of Pediatrics ( $p=0.026,0.029$ and 0.036, respectively), while the highest prevalence of levofloxacin resistance was found in other departments $(p=0.017)$. However, no significant difference was observed in the prevalence of minocycline resistance in S. maltophilia isolated from various departments of the hospital $(p>0.05)$. 
Table 2. Resistance of Stenotrophomonas maltophilia to three fluoroquinolone antibacterial drugs from 2010 to 2012.

\begin{tabular}{|c|c|c|c|c|c|c|c|c|}
\hline \multirow{2}{*}{$\begin{array}{c}\text { Fluoroquinolone } \\
\text { Antibacterial }\end{array}$} & \multicolumn{2}{|c|}{$2010(n=69)$} & \multicolumn{2}{|c|}{$2011(n=148)$} & \multicolumn{2}{|c|}{$2012(n=209)$} & \multirow{2}{*}{$\chi^{2}$ value } & \multirow{2}{*}{$p$ value } \\
\hline & Resistant (\%) & Susceptible (\%) & Resistant (\%) & Susceptible (\%) & Resistant (\%) & Susceptible (\%) & & \\
\hline Levofloxacin & 0 & 100 & 4 & 94.6 & 4.1 & 93 & 3.102 & 0.212 \\
\hline Sulfamethoxazole & 53 & 47 & 66 & 34 & 86.6 & 13.4 & 36.963 & 0 \\
\hline
\end{tabular}

Table 3. Resistance to three fluoroquinolone antibacterial drugs in Stenotrophomonas maltophilia isolated from various departments of the hospital.

\begin{tabular}{|c|c|c|c|c|c|c|c|}
\hline \multirow{2}{*}{ Department } & \multirow{2}{*}{ No. Bacterial Isolate } & \multicolumn{2}{|c|}{ Levofloxacin } & \multicolumn{2}{|c|}{ Sulfamethoxazole } & \multicolumn{2}{|c|}{ Minocycline } \\
\hline & & Resistant (\%) & Susceptible (\%) & Resistant (\%) & Susceptible (\%) & Resistant (\%) & Susceptible (\%) \\
\hline ICU & 113 & 2.7 & 97.3 & 72.1 & 27.9 & 0.9 & 98.2 \\
\hline Respiratory medicine & 82 & 6.3 & 92.4 & 72.2 & 27.8 & 1.3 & 98.7 \\
\hline Neurosurgery & 49 & 8.5 & 89.4 & 82.6 & 17.4 & 0 & 97.9 \\
\hline Pediatrics & 43 & 0 & 97.7 & 72.1 & 27.9 & 0 & 100 \\
\hline Emergency & 29 & 0 & 89.3 & 75 & 25 & 0 & 100 \\
\hline Neurology & 20 & 0 & 95 & 95 & 5 & 0 & 100 \\
\hline Others & 90 & 12.4 & 86.2 & 81.7 & 18.3 & 0 & 98.6 \\
\hline
\end{tabular}

Table 4. Resistance of Stenotrophomonas maltophilia to three fluoroquinolone antibacterial drugs among patients at 3 age groups.

\begin{tabular}{ccccccccc}
\hline \multirow{2}{*}{ Age Group (years) } & \multicolumn{2}{c}{ Bacterial Isolate } & \multicolumn{2}{c}{ Levofloxacin } & \multicolumn{2}{c}{ Sulfamethoxazole } & \multicolumn{2}{c}{ Minocycline } \\
\cline { 2 - 8 } & No. & Percentage (\%) & Resistant (\%) & Susceptible (\%) & Resistant (\%) & Susceptible (\%) & Resistant (\%) & Susceptible (\%) \\
\hline$<18$ & 59 & 13.8 & 0 & 98.6 & 76.8 & 23.2 & 0 & 100 \\
$18-59$ & 129 & 30.3 & 2.1 & 96.8 & 70.6 & 29.4 & 0.5 & 98.9 \\
$\geq 60$ & 238 & 55.9 & 6.3 & 91.2 & 77.1 & 22.9 & 0.6 & 98.2 \\
\hline
\end{tabular}


Table 5. In vitro susceptibility of Stenotrophomonas maltophilia to 7 antimicrobials ( $n=114)$.

\begin{tabular}{|c|c|c|c|c|c|c|}
\hline Antimicrobial & $\mathrm{MIC}_{\mathrm{R}}(\mu \mathrm{g} / \mathrm{mL})$ & $\mathrm{MIC}_{50}(\mu \mathrm{g} / \mathrm{mL})$ & $\mathrm{MIC}_{90}(\mu \mathrm{g} / \mathrm{mL})$ & $\begin{array}{l}\text { Percentage of } \\
\text { Susceptible Isolate (\%) }\end{array}$ & $\begin{array}{l}\text { Percentage of } \\
\text { Intermediate Isolate }(\%)\end{array}$ & $\begin{array}{l}\text { Percentage of } \\
\text { Resistant Isolate }(\%)\end{array}$ \\
\hline Norfloxacin & $0.5-128$ & 8 & 64 & 45.7 & 21.9 & 32.4 \\
\hline Ofloxacin & $0.125-64$ & 1 & 4 & 73.6 & 13.2 & 13.2 \\
\hline Chloramphenicol & $0.25-64$ & 8 & 64 & 51.7 & 23.7 & 24.6 \\
\hline Minocycline & $0.25-64$ & 1 & 4 & 90.3 & 4.4 & 5.3 \\
\hline Ceftazidime & $0.5-128$ & 8 & 128 & 66.7 & 10.5 & 22.8 \\
\hline Levofloxacin & $0.25-64$ & 2 & 8 & 73.7 & 14.0 & 12.3 \\
\hline Ciprofloxacin & $0.25-64$ & 1 & 4 & 54.4 & 23.7 & 21.9 \\
\hline
\end{tabular}

$\mathrm{MIC}_{\mathrm{R}}$, range of minimum inhibitory concentration (MIC); $\mathrm{MIC}_{50}$ : MIC required to inhibit the growth of 50\% of organisms; MIC 90 : MIC required to inhibit the growth of $90 \%$ of organisms.

Table 6. Effect of reserpine treatment on the susceptibility of 114 Stenotrophomonas maltophilia isolates to 3 fluoroquinolone antimicrobials.

\begin{tabular}{|c|c|c|c|c|c|c|c|}
\hline Antimicrobial & $\mathrm{MIC}_{\mathrm{R}}(\boldsymbol{\mu g} / \mathrm{mL})$ & $\begin{array}{c}\text { No. Resistant } \\
\text { Isolate }\end{array}$ & $\begin{array}{c}\text { Percentage of } \\
\text { Resistant Isolate (\%) }\end{array}$ & $\begin{array}{c}\text { No. Intermediate } \\
\text { Isolate }\end{array}$ & $\begin{array}{c}\text { Percentage of } \\
\text { Intermediate Isolate (\%) }\end{array}$ & $\begin{array}{c}\text { No. Susceptible } \\
\text { Isolate } \\
\end{array}$ & $\begin{array}{c}\text { Percentage of } \\
\text { Susceptible Isolate }(\%)\end{array}$ \\
\hline Norfloxacin & $0.5-128$ & 37 & 32.4 & 25 & 21.9 & 52 & 45.7 \\
\hline Norfloxacin +reserpine & $0.5-128$ & 25 & 21.9 & 19 & 16.7 & 70 & 61.4 \\
\hline Ciprofloxacin & $0.25-64$ & 25 & 21.9 & 27 & 23.7 & 62 & 54.4 \\
\hline Ciprofloxacin + reserpine & $0.25-64$ & 15 & 13.2 & 22 & 19.3 & 77 & 67.5 \\
\hline Ofloxacin & $0.125-64$ & 15 & 13.2 & 15 & 13.2 & 84 & 73.6 \\
\hline Ofloxacin + reserpine & $0.125-64$ & 11 & 9.6 & 11 & 9.6 & 92 & 80.8 \\
\hline
\end{tabular}

Table 7. Comparison of fluoroquinolone drug resistance phenotype between efflux pump-positive and -negative isolates of Stenotrophomonas maltophilia.

\begin{tabular}{ccccccc}
\hline \multirow{2}{*}{ Antimicrobial } & \multicolumn{2}{c}{ Efflux Pump-Positive Isolate $(\boldsymbol{n}=\mathbf{1 9})$} & \multicolumn{2}{c}{ Efflux Pump-Negative Isolate $(\boldsymbol{n}=\mathbf{9 5})$} \\
\cline { 2 - 7 } & Resistant (\%) & Intermediate (\%) & Susceptible (\%) & Resistant (\%) & Intermediate (\%) & Susceptible (\%) \\
\hline Norfloxacin & $18(94.7)$ & 0 & $1(5.3)$ & $19(20)$ & $25(26.3)$ & $51(53.7)$ \\
Ciprofloxacin & $16(94.2)$ & $1(5.3)$ & $2(10.5)$ & $9(9.5)$ & $26(27.4)$ & $60(63.1)$ \\
Ofloxacin & $15(78.9)$ & $3(15.8)$ & $1(5.3)$ & 0 & $12(12.6)$ & $83(87.4)$ \\
\hline
\end{tabular}




\subsection{Fluoroquinolone Resistance in S. maltophilia Isolated from Patients at Various Age Groups}

No levofloxacin resistance was detected in the $S$. maltophilia isolated from patients aged $<18$ years, and the prevalence of levofloxacin resistance was higher in S. maltophilia isolated from patients aged $\geq 60$ years than from patients aged $<18$ years $(p=0.046)$. A high prevalence of sulfamethoxazole resistance and low prevalence of minocycline resistance were observed in S. maltophilia clinical isolates (Table 4).

\subsection{Effect of Reserpine on MIC of S. maltophilia to Fluoroquinolone}

The MIC of norfloxacin, ofloxacin, chloramphenicol, minocycline, ceftazidime, levofloxacin, and ciprofloxacin on the 114 S. maltophilia clinical isolates from 2012 was shown in Table 5. All the 114clinical isolates of $S$. maltophilia grew well on the plate of $\mathrm{M}-\mathrm{H}$ agar containing reserpine. There were two, 10 and $11 \mathrm{~S}$. maltophilia clinical isolates with reduction in the MIC of norfloxacin, ciprofloxacin, and ofloxacin by $3 / 4$ or more following reserpine treatment, respectively. These bacterial isolates were defined as pump positive; however, $3 / 4$ or higher reduction in MIC of two fluoroquinolones was found in four isolates. Finally, a total of 19 efflux pump-positive S. maltophilia clinical isolates were identified. Our findings showed significant differences in the sensitivity of $S$. maltophilia to norfloxacin $\left(\chi^{2}=4.788, p<0.05\right)$ and ciprofloxacin $\left(\chi^{2}=3.982, p<0.05\right)$ before and after reserpine treatment, while no significant difference was detected in the susceptibility of $S$. maltophilia to ofloxacin $\left(\chi^{2}=0.9\right.$, $p>0.05$ ) (Table 6).

\subsection{Association of Efflux Pump Phenotype with Resistance of S. maltophilia to Fluoroquinolone}

The efflux pump-negative $S$. maltophilia isolates showed significantly greater susceptibility than efflux pump-negative isolates to norfloxacin, ciprofloxacin, and ofloxacin $\left(\chi^{2}=7.326,6.904\right.$ and 12.756, all $p$ values $<0.05)$ (Table 7).

\subsection{Prevalence of Fluoroquinolone Resistance Genes}

gyrA, parC, smeD, smeE and smeF genes were detected in all 114 clinical isolates of S. maltophilia, with $100 \%$ prevalence seen, and the prevalence of smqnr gene was $25.4 \%$; however, no other fluoroquinolone resistance genes were detected.

\section{8. mRNA Expression of smeD and smeF in S. maltophilia}

High expression of $s m e D$ and $s m e F \quad m R N A$ was detected in two efflux pump-positive, fluoroquinolone-resistant isolates of $S$. maltophilia (R100 and R106 isolates), while relatively low expression was observed in two efflux pump-negative, fluoroquinolone-susceptible isolates (S74 and S128 isolates) and one efflux pump-positive, fluoroquinolone-resistant isolate of $S$. maltophilia (R25) (Figure 1). The relative $m R N A$ expression of $s m e D$ and $s m e F$ genes was $3.755 \pm 1.506$ and $28.335 \pm 31.431$ in efflux pump-positive $S$. maltophiliaisolates, which was significantly higher than that $(1.170 \pm 0.416$ and $1.757 \pm 0.518)$ in efflux pump-negative isolates $(t=4.384$ and 44.443 , both $p$ values $<0.05)$. 

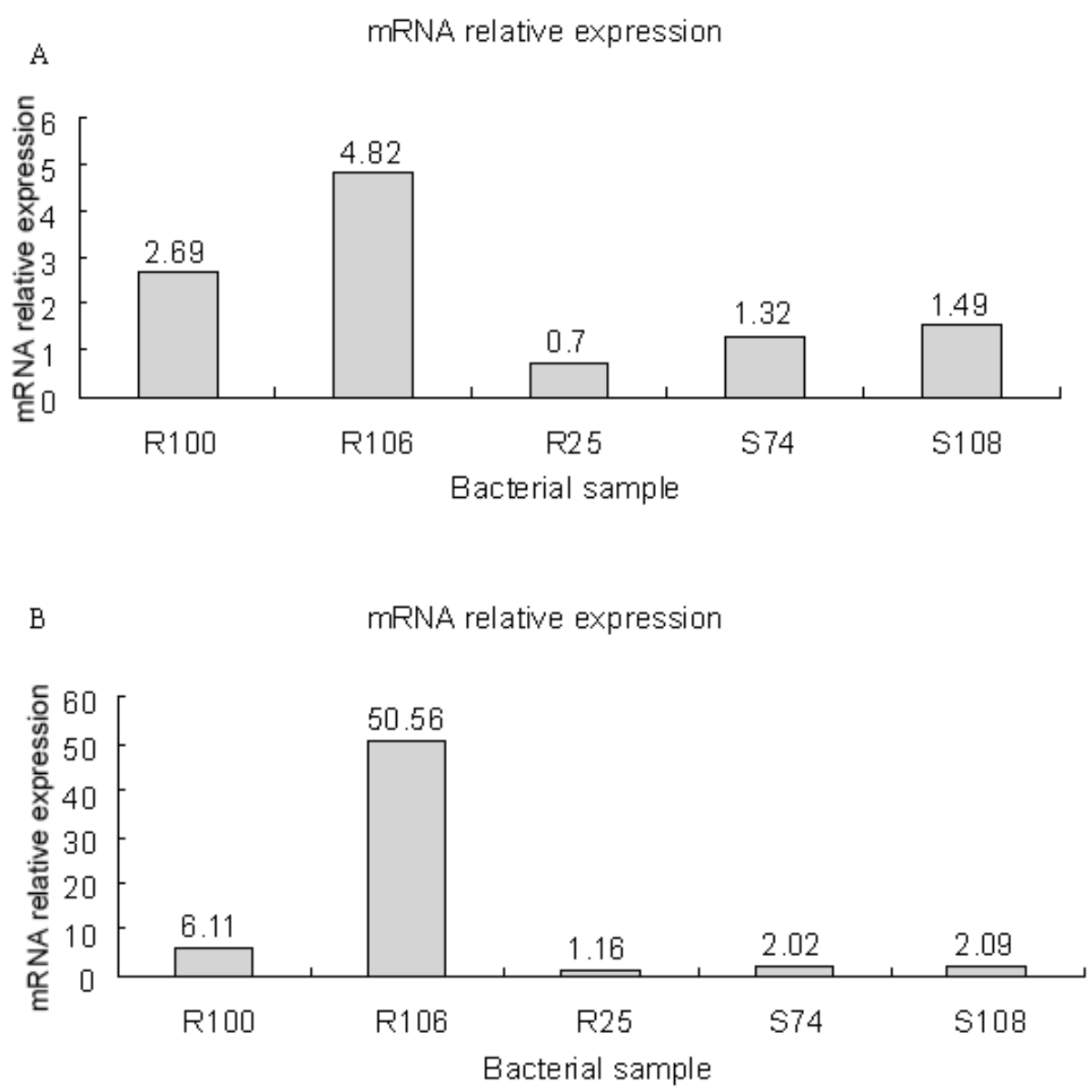

Figure 1. Relative mRNA expression of $s m e D(\mathbf{A})$ and $s m e F(\mathbf{B})$ genes.

\subsection{Sequence Analysis of gyrA, parC and smqnr Genes}

gyrA (300 bp in length) and parC (273 bp in length) genes were detected in 95 efflux pump-negative isolates of $S$. maltophilia. The PCR products of the gyrA and parC genes extracted from fiveS. Maltophilia isolates were sequenced and then aligned with the sequence of the control ATCC13637 strain. Our findings showed Glu-Lys mutation (GAA-AAA) was detected at the 151th amino acid of the gyrA gene from two fluoroquinolone-resistant isolate of S. maltophilia (R101 and R138) and one fluoroquinolone-susceptible isolate (S62), while Gly-Arg mutation (GGC-CGC) was found at the 37th amino acid of the $\operatorname{parC}$ gene derived from two fluoroquinolone-resistant isolate of S. maltophilia (R101 and R138) and two fluoroquinolone-susceptible isolate (S31 and S126) (Table 8). There were no significant differences in the prevalence of mutation at 151th amino acid of the gyrA gene $(p=0.4)$ and at the 37 th amino acid of the parC gene $(p=1)$ between the fluoroquinolone-susceptible and -resistant $S$. maltophilia isolates.

The smqnr gene was amplified from 14 randomly selected $S$. maltophilia isolates, sequenced, and aligned to the gene sequences of 58 smqnr variants accessed in GenBank. The sequence of the smqnr gene amplified from four isolates was completely consistent with the sequence of smqnr11 gene (GenBank accession number: AB430848), that from three isolates completely consistent with the sequence of smqnr35 gene (GenBank accession number: HQ896263), that from one isolate completely consistent with the sequence of smqnr8 gene (GenBank accession number: AB430850), that from one isolate completely 
consistent with the sequence of smqnr9 gene (GenBank accession number: AB430846), that from one isolate completely consistent with the sequence of smqnr4l gene (GenBank accession number: HQ896273), and that from one isolate completely consistent with the sequence of smqnr53 gene (GenBank accession number: AB852573). After sequence alignment between two fluoroquinolone resistant gene mutant isolates and smqnrl gene (GenBank accession number: AB430839), there were seven amino acid mutations found in S. maltophilia isolate 90, and 11 amino acid mutations found in S. maltophilia isolate 116. There were one or more differences in the amino acid sequence among the smqnr genes amplified from 14 S. maltophilia clinical isolates, with $92 \%-99 \%$ heterogenicity.

\subsection{0. $P F G E$}

PFGE of 29 smqnr gene-positive S. maltophilia clinical isolates revealed 25 genotypes and 28 subgenotypes (Figure 2).

Isolates numbered 28 and 62 shared the same PFGE type, isolates numbered 70 and 91 showed two PFGE subgenotypes, with 1- to 3-band difference, and isolates 80, 138 and 141showed three PFGE subgenotypes, with 1- to 3-band difference.

\section{Discussion}

S. maltophilia, a non-fermentative bacterium, Gram-negative, conditionally pathogenic bacterium that frequently colonizes the human respiratory tract and intestinal tract, may induce pneumonia, meningitis and chronic enteritis [1-3].In this study, a total of 426 S. maltophilia strains were isolated from auniversity hospital in Northwestern China during the period from 2010 through 2012, which accounted for $10.1 \%(426 / 4,225)$ of total non-fermentative bacteria (it ranks third) and $1.8 \%$ $(426 / 23,880)$ of total clinical bacterial isolates, and the prevalence of $S$. maltophilia ranked sixth among all Gram-negative bacilli. In addition, the majority of $S$. maltophilia strains were isolated from the respiratory tract specimens collected from the ICU, department of Respiratory Medicine and Department of Neurosurgery, and $55.9 \%$ of the S. maltophilia strains were isolated from patients aged over 60, suggesting that elderly patients are at high risk of $S$. maltophilia infections. Due to its inherent resistance to many antimicrobials, the antimicrobial susceptibility testing of $S$. maltophilia is of great importance to guide clinical antibiotic use [34]. Among the $426 \mathrm{~S}$. maltophilia clinical isolates, the prevalence of levofloxacin, sulfamethoxazole and minocycline resistance was $3.3 \%, 74.3 \%$ and $0.5 \%$, respectively, and a gradually increasing tendency was found in the prevalence of antimicrobial resistance in $S$. maltophilia year by year with the extensive use of antimicrobials. 
Table 8. Amino acid mutation in gyrA and parC genes of 5Stenotrophomonas maltophilia isolates and MIC of three fluoroquinolone agents.

\begin{tabular}{|c|c|c|c|c|c|c|c|c|}
\hline \multirow{2}{*}{ Bacteria Number } & \multicolumn{3}{|c|}{ Antimicrobial MIC $(\mu \mathrm{g} / \mathrm{mL})$} & \multicolumn{3}{|l|}{ gyrA } & \multicolumn{2}{|l|}{$\overline{p a r C}$} \\
\hline & Norfloxacin & Ciprofloxacin & Ofloxacin & 85th amino acid & 119th amino acid & 151th amino acid & 37th amino acid & 72th amino acid \\
\hline ATCC13637 & 0.5 & 0.25 & 0.25 & Valine (GTC) & Alanine (GCA) & Glutamic acid (GAA) & Glycin (GGC) & Glycin (GGT) \\
\hline S31 & 4 & 0.5 & 0.5 & Valine (GTG) & Alanine (GCG) & - & Arginine (CGC) & - \\
\hline S62 & 2 & 1 & 1 & Valine (GTG) & Alanine (GCG) & Lysine (AAA) & - & - \\
\hline R101 & 32 & 8 & 16 & Valine (GTG) & Alanine (GCG) & Lysine (AAA) & Arginine (CGC) & - \\
\hline S126 & 8 & 2 & 2 & Valine (GTG) & Alanine (GCG) & - & Arginine (CGC) & Glycin (GGC) \\
\hline R138 & 32 & 4 & 8 & Valine (GTG) & - & Lysine (AAA) & Arginine (CGC) & Glycin (GGC) \\
\hline
\end{tabular}

S31, S62 and S126 were fluoroquinolone-susceptible Stenotrophomonas maltophilia isolates; R101 and R138 were fluoroquinolone resistant.

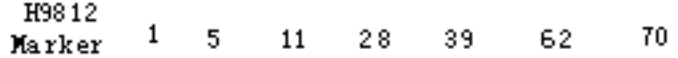

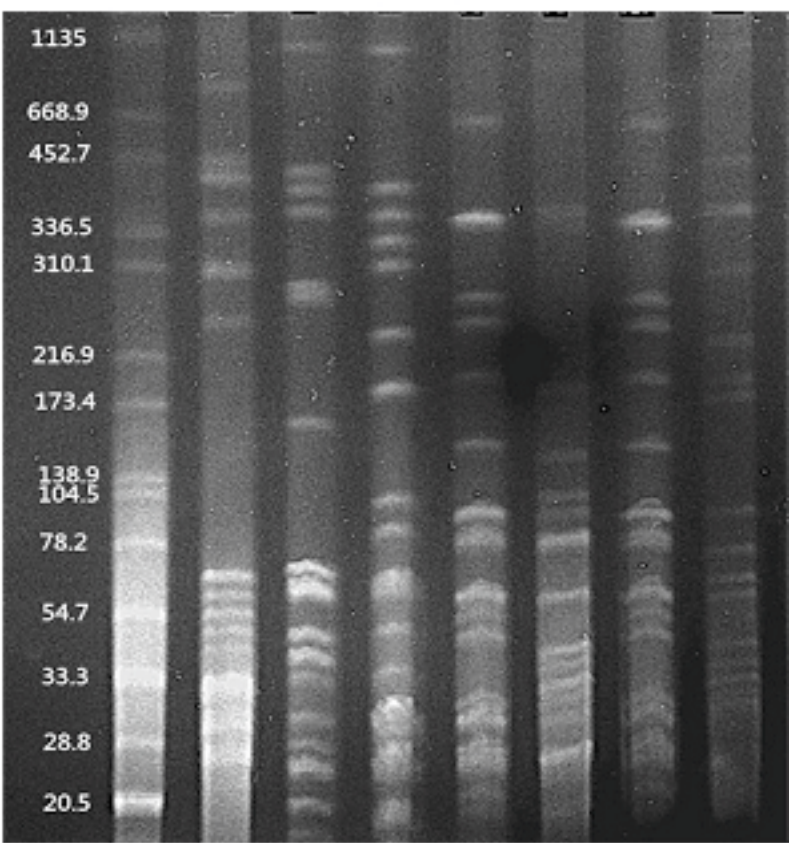

Figure 2. PFGE of 29 smqnr gene-positive S. maltophilia clinical isolates. 
The efflux pump inhibitor reserpine, an indole alkaloid, can block the substrate across the efflux channel, thereby increasing the intracellular drug concentrations [35]. Due to low specificity and affinity of reserpine-membrane glycoprotein binding, a high dose of these two drugs is required to achieve the inhibition; however, the dose that achieves inhibition on activity of efflux pump may induce neurotoxicity [36,37], which limits their clinical application. Our findings showed that reserpine inhibited the pumping out of fluoroquinolones in some $S$. maltophilia clinical isolates, resulting in a reduced prevalence of antimicrobial resistance. In this study, a total of 38 fluoroquinolone-resistant and 76 fluoroquinolone-susceptible $S$. maltophilia isolates were screened, and a significant difference was detected in the prevalence of efflux pump between the fluoroquinolone-resistant $(18 / 38)$ and fluoroquinolone-susceptible bacterial isolates $(1 / 76)\left(\chi^{2}=25.189, p<0.05\right)$. These results demonstrated the efflux ability in both fluoroquinolone-resistant and -susceptible $S$. maltophilia isolates, and a higher activity was found in resistant isolates, indicating that efflux pump increases the resistance to $S$. maltophilia to fluoroquinolones. However, we did not identify S. maltophilia clinical isolates positive for efflux pump of all three fluoroquinolones, including norfloxacin, ciprofloxacin, and ofloxacin, indicating that fluoroquinolones have various efflux capabilities due to different biochemical characteristics caused by the structural difference.

Mutations of DNA gyrase and topoisomerase [15-18], mutation of drug resistance genes [19,20] and drug efflux pumps [21-23] are reported to be involved in the emergence of antibiotic resistance in S. maltophilia. Efflux pump system has been proved to be the most important cause responsible for the development of intrinsic and acquired multidrug resistance in S. maltophilia [20]. SmeABC multidrug efflux pump system is the first efflux pump gene identified in S. maltophilia [32], which is homogenous to the MexAB-OprM efflux pump system in Pseudomonas aeruginosa [38]. SmeDEF, a root nodulation and division (RND) family member, is an efflux pump system associated with multidrug resistance in S. maltophilia [39]. The substrates of SmeDEF include fluoroquinolones, macrolides and tetracyclines, which are mutually different in structures [40]. In the current study, we detected high mRNA expression of smeD and smeF genes in two efflux pump-positive, fluoroquinolone-resistant $S$. maltophilia isolates, and low expression in two efflux pump-negative, fluoroquinolone-susceptible isolates and one efflux pump-negative, fluoroquinolone-resistant isolate. These findings are consistent with those resulting from previous studies $[41,42]$, indicating that smeD and smeF play vital roles in the development of resistance of $S$. maltophilia to fluoroquinolones.

Two enzymes, DNA gyrase and topoisomerase IV, are major targets of fluoroquinolones[43-45]. DNA gyrase consists of two subunits, GyrA and GyrB [46], and topoisomerase IV is composed of two ParC subunits (homogenous to GyrA) and two ParE subunits (homogenous to GyrB) [16]. In most gram-negative bacteria, gyrAis the primary target conferring fluoroquinolone resistance, $\operatorname{par} C$ as the secondary target, and the alteration of $g y r B$ and pare expression is found to improve drug resistance level [47]. Therefore, gyrA and $\operatorname{parC}$ genes were amplified from three randomly selected fluoroquinolone-susceptible $S$. maltophilia clinical isolates and two randomly selected fluoroquinolone-resistant isolates and sequenced; however, we did not detect mutations associated with fluoroquinolone resistance that are frequently identified in other Gram-negative bacteria, such as mutations in the Ser-83 codons and Asp-87 codons of the gyrA gene and Gly-84 codons of the $\operatorname{par} C$ gene. In addition, there was significant difference found in the prevalence of $g y r A$ and $\operatorname{par} C$ mutation between fluoroquinolone-susceptible and -resistant $S$. maltophilia, indicating that the mutation 
in the gyrA and parC genes did not correlate with fluoroquinolone resistance in S. maltophilia. Sequencing revealed almost identical nucleic acid sequences of gyrA and $\operatorname{parC}$ genes between fluoroquinolone-susceptible and -resistant isolates, which further demonstrated that the resistance of S. maltophilia to fluoroquinolones was not associated with targeted gene mutation. Such a finding was consistent with the results from the study Ribera et al. [48], but inconsistent with the study by Liu and colleagues [50]. Ribera and colleagues [48] reported no amino acid changes in the quinolone resistance-determining region (QRDR) of either gyrA or parC genes among quinolone-susceptible and -resistant $S$. maltophilia strains, and concluded that the development of resistance to quinolones was not related to mutations in the QRDR of gyrA and parC genes in the $S$. maltophilia isolates, contrary to what has been described in other microorganisms. Liu et al. [49] found an increase in the MIC of fluoroquinolones for $S$. maltophilia isolates with mutations in the $\operatorname{gyr} A$ or $\operatorname{parC}$ genes $(8-16 \mu \mathrm{g} / \mathrm{L})$ as compared to the isolates without mutations in the gyrA or parC genes $(2-4 \mu \mathrm{g} / \mathrm{L})$, and detected mutation at the 80th amino acid of the $\operatorname{parC}$ gene in one isolate, leading to substitution of serine by isoleucine (AGC-ATC), and at the 57th amino acid of the gyr $A$ gene leading to substitution of alanine by arginine (GCG-CGC/CGG) and at the 44th amino acid of the parC gene (GGC-CGC) leading to substitution of glycine by arginine in two isolates, demonstrating that the mutation of gyrA and parC genes may be associated with the resistance to fluoroquinolone in $S$. maltophilia.

Plasmid-mediated quinolone resistance (PMQR) is a newly identified mechanism of antimicrobial resistance, and it is mainly mediated by antibiotic resistance $q n r$ gene, including subtypes of $q n r A, q n r B$, $q n r C, q n r D$ and $q n r S$, in which $q n r A$ is the most common subtype [50]. PMQR is frequently detected in Enterobacteriaceae [51,52], and it has been detected in non-fermentative P. aeruginosa [53]. However, no PMQR is reported in S. maltophilia till now, and PMQR genes were not detected in the S. maltophilia clinical isolates identified in the present study.

It has been recently shown that $S$. maltophilia contains a chromosomally encoded qnr gene (Smqnr) which confers low-level resistance to fluoroquinolones [54]. Smqnr is a new quinolone resistance gene locating in the chromosome in S. maltophilia, which was firstly identified in 2008 [55]. There have been 58 smqnr genes identified so far, and only variations in several amino acids are found among these smqnr genes. In this study, the prevalence of smqnr gene was $25.4 \%$ in the 114 S. maltophilia clinical isolates included in this study.

Since $S$. maltophilia is considered a hospital-acquired colonizing bacterium, it does not receive much attention. However, S. maltophilia infections may cause high mortality. The spread of S. maltophilia isolates across a hospital would greatly threaten inpatients' health. PFGE is accepted as the gold standard for genotyping bacteria due to high resolution and repeatability [56]. In this study, PFGE assay was employed for molecular identification of $S$. maltophilia clinical isolates, and 25 PFGE genotypes and 28 subgenotypes were detected in the 29 S. maltophilia clinical isolates; however, the predominant colony could not be identified due to the limited number of bacterial isolates with the same subgenotype.

\section{Conclusions}

S. maltophilia is an important hospital-acquired pathogenic bacterium, and sterilization and isolation of patients should be strengthened due to multidrug antimicrobial resistance and clonal spread of 
S. maltophilia clinical isolates. Monitoring the clinical distribution and antimicrobial resistance of S. maltophilia is of great significance for the clinical therapy of the bacterial infection. Reserpine is effective atinhibiting the active efflux of norfloxacin, ciprofloxacin and ofloxacinin S. maltophilia and reducing theMIC of fluoroquinolones against the bacteria. The resistance of S. maltophilia to fluoroquinolones shows no significant association with the alteration in the targeted site of DNA gyrase and topoisomerase IV, while the expression of efflux pump smeD and smeF genes correlates with the resistance of $S$. maltophilia to fluoroquinolones.

\section{Acknowledgements}

Many thanks are addressed to all participants for their kind cooperation. This study was supported by the grant from Ningxia Science and Technology Project.

\section{Author Contributions}

Wei Jia conceived and designed the study; Jiayuan Wang, Haotong Xu and Gang Li conducted the study, collected the data and performed analysis of data. Jiayuan Wang prepared the first draft of the manuscript; Wei Jia provided strategic advice and assisted with editing of the manuscript. All authors read and approved the final version of the manuscript.

\section{Conflicts of Interest}

The authors declare no conflict of interest.

\section{References}

1. Looney, W.J.; Narita, M.; Mühlemann, K. Stenotrophomonas maltophilia: An emerging opportunist human pathogen. Lancet Infect. Dis. 2009, 9, 312-323.

2. Brooke, J.S. Stenotrophomonas maltophilia: An emerging global opportunistic pathogen. Clin.Microbiol. Res.2012, 25, 2-41.

3. Palleroni, N.J.; Bradbury, J.F. Stenotrophomonas, a new bacterial genus for Xanthomonas maltophilia (Hugh 1980) Swings et al. 1983. Int. J. Syst. Bacteriol. 1993, 43, 606-609.

4. Cheong, H.S.; Lee, J.A.; Kang, C.I.; Chung, D.R.; Peck, K.R.; Kim, E.S.; Lee, J.S.; Son, J.S.; Lee, N.Y.; Song, J.H. Risk factors for mortality and clinical implications of catheter-related infections in patients with bacteraemia caused by Stenotrophomonas maltophilia. Int. J.Antimicrob. Agents 2008, 32, 538-540.

5. Kara, I.H.; Yilmaz, M.E.; Sit, D.; Kadiroğlu, A.K.; Kökoğlu, O.F. Bacteremia caused by Stenotrophomonas maltophilia in a dialysis patient with a long-term central venous catheter. Infect. Control Hosp. Epidemiol. 2006, 27, 535-536.

6. Abbott, I.J.; Slavin, M.A.; Turnidge, J.D.; Thursky, K.A.; Worth, L.J. Stenotrophomonas maltophilia: Emerging disease patterns and challenges for treatment. Expert Rev. Anti Infect.Ther.2011, 9, 471-488.

7. Nicodemo, A.C.; Paez, J.I. Antimicrobial therapy for Stenotrophomonas maltophilia infections. Eur. J.Clin. Microbiol. Infect. Dis. 2007, 26, 229-237. 
8. Waters, V. New treatments for emerging cystic fibrosis pathogens other than Pseudomonas. Curr. Pharm. Des.2012, 18, 696-725.

9. Bellido, J.L.; Hernández, F.J.; Zufiaurre, M.N.; García-Rodríguez, J.A. In vitro activity of newer fluoroquinolones against Stenotrophomonas maltophilia. J.Antimicrob.Chemother.2000, 46, 334-335.

10. Schmitz, F.J.; Verhoef, J.; Fluit, A.C. Comparative activities of six different fluoroquinolones against 9,682 clinical bacterial isolates from 20 European university hospitals participating in the European SENTRY surveillance programme. The SENTRY participants group. Int. J. Antimicrob. Agents1999, 12, 311-317.

11. Isenberg, H.D.; Alperstein, P.; France, K. In vitro activity of ciprofloxacin, levofloxacin, and trovafloxacin, alone and in combination with beta-lactams, against clinical isolates of Pseudomonas aeruginosa, Stenotrophomonas maltophilia, and Burkholderia cepacia. Diagn.Microbiol. Infect. Dis. 1999, 33, 81-86.

12. TrigoDaporta, M.; Muñoz Bellido, J.L.; García-Rodríguez, J.A. Topoisomerases mutations and fluoroquinolone resistance in Stenotrophomonas maltophilia. Int. J. Antimicrob. Agents2004, 24, $520-521$.

13. Garrison, M.W.; Anderson, D.E.; Campbell, D.M.; Carroll, K.C.; Malone, C.L.; Anderson, J.D.; Hollis, R.J.; Pfaller, M.A. Stenotrophomonas maltophilia: Emergence of multidrug-resistant strains during therapy and in an in vitro pharmacodynamic chamber model. Antimicrob. Agents Chemother.1996, 40, 2859-2864.

14. Baek, J.H.; Kim, C.O.; Jeong, S.J.; Ku, N.S.; Han, S.H.; Choi, J.Y.; Yong, D.; Song, Y.G.; Lee, K.; Kim, J.M. Clinical factors associated with acquisition of resistance to levofloxacin in Stenotrophomonas maltophilia. Yonsei Med. J.2014, 55, 987-993.

15. Oizumi, N.; Kawabata, S.; Hirao, M.; Watanabe, K.; Okuno, S.; Fujiwara, T.; Kikuchi, M. Relationship between mutations in the DNA gyrase and topoisomerase IV genes and nadifloxacin resistance in clinically isolated quinolone-resistant Staphylococcus aureus. J. Infect.Chemother.2001, 7, 191-194.

16. Drlica, K.; Zhao, X. DNA gyrase, topoisomerase IV, and the 4-quinolones. Microbiol. Mol. Biol. Rev. 1997, 61, 377-392.

17. Vranakis, I.; Sandalakis, V.; Chochlakis, D.; Tselentis, Y.; Psaroulaki, A. DNA gyrase and topoisomerase IV mutations in an in vitro fluoroquinolone-resistant Coxiella burnetii strain. Microb. Drug Resist. 2010, 16, 111-117.

18. Weigel, L.M.; Anderson, G.J.; Tenover, F.C. DNA gyrase and topoisomerase IV mutations associated with fluoroquinolone resistance in Proteus mirabilis. Antimicrob. Agents Chemother.2002, 46, 2582-2587.

19. García-León, G.; Salgado, F.; Oliveros, J.C.; Sánchez, M.B.; Martínez, J.L. Interplay between intrinsic and acquired resistance to quinolones in Stenotrophomonas maltophilia. Environ.Microbiol.2014, 16, 1282-1296.

20. Gordon, N.C.; Wareham, D.W. Novel variants of the Smqnr family of quinolone resistance genes in clinical isolates of Stenotrophomonas maltophilia. J.Antimicrob.Chemother.2010, 65, 483-489.

21. Poole, K. Efflux pumps as antimicrobial resistance mechanisms. Ann. Med. 2007, 39, 162-176. 
22. Soto, S.M. Role of efflux pumps in the antibiotic resistance of bacteria embedded in a biofilm. Virulence 2013,4, 223-229.

23. Poole, K. Multidrug efflux pumps and antimicrobial resistance in Pseudomonas aeruginosa and related organisms. J. Mol. Microbiol. Biotechnol. 2001, 3, 255-264.

24. Tsaur, S.M.; Chang, S.C.; Luh, K.T.; Hsieh, W.C. Antimicrobial susceptibility of enterococci in vitro. J. Formos. Med. Assoc. 1993, 92, 547-552.

25. Valdezate, S.; Vindel, A.; Echeita, A.; Baquero, F.; Cantó, R. Topoisomerase II and IV quinolone resistance-determining regions in Stenotrophomonas maltophilia clinical isolates with different levels of quinolone susceptibility. Antimicrob. Agents Chemother. 2002, 46, 665-671.

26. Sun, E.L.; Song, S.D.; Wei, D.J. Effect of efflux inhibitor on the activity of fluoroquinlone to Stenotrophomonas maltophilia. Chin. J.Antibiot.2003, 28, 757-760.

27. Zhao, J.Y.; Dang, H. Coastal seawater bacteria harbor a large reservoir of plasmid-mediated quinolone resistance determinants in Jiaozhou Bay, China. Microb. Ecol. 2012, 64, 187-199.

28. Gould, V.C.; Avison, M.B. SmeDEF-mediated antimicrobial drug resistance in Stenotrophomonas maltophilia clinical isolates having defined phylogenetic relationships. J. Antimicrob. Chemother. 2006, 57, 1070-1076.

29. Yuan, J.; Xu, X.; Guo, Q.; Zhao, X.; Ye, X.; Guo, Y.; Wang, M. Prevalence of the oqxAB gene complex in Klebsiella pneumonia and Escherichia coli clinical isolates. J.Antimicrob. Chemother. 2012, 67, 1655-1659.

30. Zhang, R.; Sun, Q.; Hu, Y.J.; Yu, H.; Li, Y.; Shen, Q.; Li, G.X.; Cao, J.M.; Yang, W.; Wang, Q.; et al. Detection of the Smqnr quinolone protection gene and its prevalence in clinical isolates of Stenotrophomonas maltophilia in China. J. Med. Microbiol. 2012, 61, 535-539.

31. Alonso, A.; Martínez, J.L. Cloning and characterization of SmeDEF, a novel multidrug efflux pump from Stenotrophomonas maltophilia. Antimicrob. Agents Chemother. 2000, 44, 3079-3086.

32. Chang, L.L.; Chen, H.F.; Chang, C.Y.; Lee, T.M.; Wu, W.J. Contribution of integrons, and $S m e A B C$ and $S m e D E F$ efflux pumps to multidrug resistance in clinical isolates of Stenotrophomonas maltophilia. J. Antimicrob. Chemother. 2004, 53, 518-521.

33. Tenover, F.C.;Arbeit, R.D.; Goering, R.V.;Mickelsen, P.A.; Murray, B.E.; Persing, D.H.; Swaminathan, B. Interpreting chromosomal DNA restriction patterns produced by pulsed-field gel electrophoresis: Criteria for bacterial strain typing. J.Clin.Microbiol.1995, 33, 2233-2239.

34. Falagas, M.E.; Valkimadi, P.E.; Huang, Y.T.; Matthaiou, D.K.; Hsueh, P.R. Therapeutic options for Stenotrophomonas maltophilia infections beyond co-trimoxazole: A systematic review. J. Antimicrob. Chemother. 2008, 62, 889-894.

35. Ughachukwu, P.O.; Unekwe, P.C. Efflux pump-mediated resistance in chemotherapy. Ann. Med. Health Sci. Res. 2012, 2, 191-198.

36. Gibbons, S.; Udo, E.E. The effect of reserpine, a modulator of multidrug efflux pumps, on the in vitro activity of tetracycline against clinical isolates of methicillin resistant Staphylococcus aureus (MRSA) possessing the tet(K) determinant. Phytother. Res. 2000, 14, 139-140.

37. Schmitz, F.J.; Fluit, A.C.; Lückefahr, M.; Engler, B.; Hofmann, B.; Verhoef, J.; Heinz, H.P.; Hadding, U.; Jones, M.E. The effect of reserpine, an inhibitor of multidrug efflux pumps, on the in vitro activities of ciprofloxacin, sparfloxacin and moxifloxacin against clinical isolates of Staphylococcus aureus. J. Antimicrob. Chemother. 1998, 42, 807-810. 
38. Li, X.Z.; Zhang, L.; Poole, K. SmeC, an outer membrane multidrug efflux protein of Stenotrophomonas maltophilia. Antimicrob. Agents Chemother. 2002, 46, 333-343.

39. Zhang, L.; Li, X.Z.; Poole, K. SmeDEF multidrug efflux pump contributes to intrinsic multidrug resistance in Stenotrophomonas maltophilia. Antimicrob. Agents Chemother. 2001, 45, 3497-3503.

40. Li, X.Z.; Nikaido, H. Efflux-mediated drug resistance in bacteria: An update. Drugs2009, 69, $1555-1623$.

41. Alonso, A.; Martinez, J.L. Expression of multidrug efflux pump SmeDEF by clinical isolates of Stenotrophomonas maltophilia. Antimicrob. Agents Chemother. 2001, 45, 1879-1881.

42. Sun, E.L.; Song, S.D.; Qi, W.; Guo, Q.L. Stenotrophomonas maltophilia SmeDEF efflux pump and the regulation mechanism. Chin. J.Microbiol.Immunol.2004, 21, 743-747.

43. Zhao, X.; Xu, C.; Domagala, J.;Drlica, K. DNA topoisomerase targets of the fluoroquinolones: A strategy for avoiding bacterial resistance. Proc. Natl. Acad. Sci. USA1997, 94, 13991-13996.

44. Chen, C.R.; Malik, M.; Snyder, M.; Drlica, K. DNA gyrase and topoisomerase IV on the bacterial chromosome: Quinolone-induced DNA cleavage. J. Mol. Biol. 1996, 258, 627-637.

45. Bearden, D.T.; Danziger, L.H. Mechanism of action of and resistance to quinolones. Pharmacotherapy2001, 21, S224-S232.

46. Reece, R.J.; Maxwell, A. DNA gyrase: Structure and function. Crit. Rev.Biochem. Mol. Biol. 1991, 26, $335-375$.

47. Weigel, L.M.; Steward, C.D.; Tenover, F.C. gyrA mutations associated with fluoroquinolone resistance in eight species of Enterobacteriaceae. Antimicrob. Agents Chemother. 1998, 42, 2661-2667.

48. Ribera, A.; Doménech-Sanchez, A.; Ruiz, J.; Benedi, V.J.; Jimenez de Anta, M.T.; Vila, J. Mutations in gyrA and parC QRDRs are not relevant for quinolone resistance in epidemiological unrelated Stenotrophomonas maltophilia clinical isolates. Microb. Drug Resist. 2002, 8, 245-251.

49. Liu, C.; Wang, Z.X.; Yao, J.; Luo, B. Relationship between gyrA and parC gene and Stenotrophomonas maltophilia to fluoroquinolone resistance. Acta Univ. Med. Anhui2011, 46, 209-212.

50. Wang, M.; Sahm, D.F.; Jacoby, G.A.; Hooper, D.C. Emerging plasmid-mediated quinolone resistance associated with the qnr gene in Klebsiella pneumoniae clinical isolates in the United States. Antimicrob. Agents Chemother. 2004, 48, 1295-1299.

51. Silva-Sánchez, J.; Cruz-Trujillo, E.; Barrios, H.; Reyna-Flores, F.; Sánchez-Pérez, A.; Bacterial Resistance Consortium.; Garza-Ramos, U. Characterization of plasmid-mediated quinolone resistance $(\mathrm{PMQR})$ genes in extended-spectrum $\beta$-lactamase-producing Enterobacteriaceae pediatric clinical isolates in Mexico. PLoS ONE 2013, 8, e 77968.

52. Cruz, G.R.; Radice, M.; Sennati, S.; Pallecchi, L.; Rossolini, G.M.; Gutkind, G.; Conza, J.A. Prevalence of plasmid-mediated quinolone resistance determinants among oxyimino cephalosporin-resistant Enterobacteriaceae in Argentina. Mem. Inst. Oswaldo Cruz 2013, 108, 924-927.

53. Jiang, X.; Yu, T.; Jiang, X.; Zhang, W.; Zhang, L.; Ma, J. Emergence of plasmid-mediated quinolone resistance genes in clinical isolates of Acinetobacter baumannii and Pseudomonas aeruginosa in Henan, China. Diagn. Microbiol. Infect. Dis. 2014, 79, 381-383. 
54. Sánchez, M.B.; Martínez, J.L. SmQnr contributes to intrinsic resistance to quinolones in Stenotrophomonas maltophilia. Antimicrob. Agents. Chemother. 2010, 54, 580-581.

55. Shimizu, K.; Kikuchi, K.; Sasaki, T.; Takahashi, N.; Ohtsuka, M.; Ono, Y.; Hiramatsu, K. Smqnr, a new chromosome-carried quinolone resistance gene in Stenotrophomonas maltophilia. Antimicrob. Agents. Chemother. 2008, 52, 3823-3825.

56. Goering, R.V. Pulsed field gel electrophoresis: A review of application and interpretation in the molecular epidemiology of infectious disease. Infect. Genet. Evol. 2010, 10, 866-875.

(C) 2015 by the authors; licensee MDPI, Basel, Switzerland. This article is an open access article distributed under the terms and conditions of the Creative Commons Attribution license (http://creativecommons.org/licenses/by/4.0/). 